\title{
Evaluación de la Calidad Docente Universitaria desde la Perspectiva de los Estudiantes
}

\section{Assessment of University Teaching Quality from the Perspective of Students}

\author{
Mariana Soledad Seivane * y María Elena Brenlla \\ Centro de Investigaciones en Psicología y Psicopedagogía (CIPP), Argentina
}

\section{DESCRIPTORES: \\ Evaluación \\ Calidad docente \\ Opinión del estudiante \\ Educación superior \\ Competencias docentes}

\begin{abstract}
RESUMEN:
El presente trabajo se plantea dos objetivos: a) analizar las propiedades psicométricas de fiabilidad y validez del Cuestionario de Competencias Docentes del Profesorado Universitario (CCDPU) para su aplicación en el Área Metropolitana de Buenos Aires (AMBA), y b) identificar las competencias y atributos mejores valorados por los estudiantes universitarios de Buenos Aires en la evaluación de la calidad docente. Método: Se llevó a cabo un estudio empírico de alcance descriptivo-correlacional para la validación del cuestionario y el análisis de los datos obtenidos. La muestra estuvo compuesta por 120 estudiantes universitarios (94 mujeres y 26 hombres) residentes en el AMBA. Resultados: Se observó que la mayoría de las subescalas del CCDPU obtuvieron valores de alfa superiores a .70 y todos los ítems fueron clasificados como coherentes, suficientes y representativos por el juicio de expertos. La subescala del cuestionario mejor valorada por los estudiantes fue Actitud del Profesorado, la cual incluye aspectos relacionados a creencias, actitudes y formas de ser del profesor que influyen en su labor y actuación docente. Discusión: Según los resultados obtenidos se deduce que, desde la perspectiva de los alumnos, los buenos docentes son aquellos que se muestran receptivos con sus estudiantes, buscan motivarlos, se interesan activamente por su aprendizaje y crean un clima adecuado de trabajo y participación. A su vez, se concluye que el CCDPU constituye una herramienta útil y válida para futuras investigaciones sobre el tema.
\end{abstract}

\section{KEYWORDS:}

Evaluation

Teaching quality

Student opinion

Higher education

Teaching skills

\begin{abstract}
:
This study has two objectives: a) to analyze the psychometric properties of reliability and validity of the Questionnaire of Teaching Skills of University Teachers (CCDPU); b) identify the competencies and attributes most valued by university students in Buenos Aires in the assessment of teaching quality. Method: An empirical study of descriptive-correlal scope was carried out for the validation of the questionnaire and the analysis of the data obtained. The participants were 120 university students (94 women and 26 men) from the Buenos Aires Metropolitan Area. Results: It was noted that most CCDPU subscales obtained alpha values higher than .70 and all items were classified as consistent, sufficient, and representative by expert judgement. The subscale of the questionnaire best valued by students was Attitude of Teachers, which includes aspects related to beliefs, attitudes and ways of being of the teacher that influence their work and teaching performance. Discussion: According to the results obtained it follows that, from the perspective of the students, good teachers are those who are receptive to their students, seek to motivate them, are actively interested in their learning and create an adequate climate of work and participation. In turn, it is concluded that the CCDPU is a useful and valid tool for future research on the subject.
\end{abstract}

CÓMO CITAR:

Seivane, M. S. y Brenlla, M. E. (2021). Evaluación de la calidad docente universitaria desde la perspectiva de los estudiantes. Revista Iberoamericana de Evaluación Educativa, 14(1), 35-46. https://doi.org/10.15366/riee2021.14.1.002

*Contacto: mariana_seivane@uca.edu.ar ISSN: 1989-0397

https://revistas.uam.es/riee 


\section{Introducción}

El nuevo escenario sociocultural ha promovido cambios en la organización de las universidades, así como también en la formación de sus docentes, lo cual trajo nuevos desafíos de la misma (Salazar-Gómez y Tobón, 2018). Es por ello que se hace necesario plantear una revisión crítica de la docencia universitaria. Se entiende a la docencia universitaria como una práctica educativa, es decir, un proceso de formación compuesto por acciones intencionadas y reflexivas, dadas dentro de la relación entre el docente y el estudiante (Martínez et al., 2005). Mediante ella se pretende cumplir con los propósitos formativos que habiliten a los estudiantes para el campo profesional del mundo laboral y social. Es, al mismo tiempo, una práctica social, ya que el docente y su actividad se encuentran inmersos en un contexto social, político y cultural particular que, inevitablemente, va a influir en la manera en que desarrolla su profesión.

Tradicionalmente, la práctica docente estaba basada en un modelo educativo centrado en la enseñanza y el profesor, donde el objetivo último era la transmisión de conocimientos. Así, el profesor era el eje central del proceso de enseñanza y aprendizaje, y ocupaba el lugar de único experto en el aula (Gargallo-López et al., 2017). No obstante, hoy en día, se busca que los procesos educativos respondan a un modelo centrado en el aprendizaje y en el alumno. En este sentido, el alumno toma protagonismo y es reconocido como actor importante y participativo de los procesos de enseñanza y aprendizaje (Hamer-Flores, 2013).

El modelo centrado en el aprendizaje se caracteriza por la construcción de significados por parte del estudiante a través del descubrimiento, la comprensión y aplicación del conocimiento a situaciones o problemas específicos (Tangney, 2014). Su objetivo es que el alumno, además de adquirir una serie de conocimientos, desarrolle procedimientos autónomos de pensamientos, y descubra, elabore, reinvente y haga suyo el conocimiento.

Desde esta perspectiva, el rol del profesor es acompañar al alumnado en la estimulación del análisis y la reflexión, facilitando ambos (Loureiro et al., 2016). Su tarea consiste en apoyar al estudiante y lograr que aprenda a aprender, razonando por sí mismo y desarrollando su capacidad de deducir, de relacionar y de elaborar síntesis (Monereo y Pozo, 2003). Es importante que el docente proporcione a los estudiantes instrumentos para pensar, para interrelacionar hechos y obtener conclusiones y consecuencias válidas.

Si entendemos a la docencia como una de las principales funciones de las universidades, su evaluación es tarea necesaria para mejorar la calidad de los procesos de enseñanza y aprendizaje. Siguiendo esta línea, la evaluación de la labor docente sirve al quehacer universitario en varios sentidos. López-Cámara (2014) refiere que es un instrumento técnico que permite tomar decisiones en relación a aspectos políticos, administrativos y académicos de las instituciones. En segundo lugar, es un instrumento de formación de intelectuales, de profesionales, que se terminarán desarrollando con su participación en el mundo del trabajo y en la sociedad en general. A su vez, Coppola (2012) plantea también que "proporciona a los profesores fuentes de retroalimentación que pueden tener una influencia positiva en su identidad y satisfacción personal y, en consecuencia, en la mejora de sus prácticas de enseñanza” (p.1).

Dentro del contexto de evaluación, existen diversos enfoques y métodos de recolección de información de cada competencia o aspecto que valore la calidad de la actividad docente (Martínez, 2013). Asimismo, existen diferentes fuentes de información para el proceso de evaluación docente. Una de las más utilizadas son los cuestionarios de opinión de los alumnos (Spooren et al., 2017). Este enfoque, basado en la perspectiva de los estudiantes, considera que ellos son capaces de proporcionar información válida y confiable acerca de los procesos de enseñanza y aprendizaje, y del cumplimiento de objetivos académicos por parte del profesor. Además, se ha constatado que las opiniones de los estudiantes, recogidas a través de este tipo de cuestionarios, permanecen estables a lo largo del tiempo, y son capaces de identificar aquellas dimensiones más relevantes a la hora de evaluar la calidad de la docencia universitaria (Romero-Díaz, 2014). Por su parte, Cortés y otros (2014) recomiendan el uso de estas encuestas de opinión, ya que contribuyen a mejorar las prácticas educativas, facilitan la toma de decisiones y permiten salvaguardar el control de la calidad educativa.

Existen algunas críticas con respecto a la evidencia basada en el juicio de los alumnos y recopilada mediante las encuestas de opinión estudiantil. Luna y Torquemada (2008) refieren que la utilización generalizada de los mismos puede traer algunas complicaciones: muchas no atienden los aspectos básicos que la literatura señala como esenciales para validar su empleo, los docentes suelen manifestar cierta disconformidad con su utilización y se suele sobrestimar o minimizar su eficacia. Por ello es requisito necesario demostrar la credibilidad de las conclusiones de estos cuestionarios. 
La búsqueda de las dimensiones y variables asociadas con la competencia docente, desde la visión de los alumnos, ha sido fuente de diversas investigaciones. Dichos estudios se esforzaron por reconocer el valor de las opiniones de los estudiantes universitarios a la hora de evaluar la labor docente. Acevedo-Álvarez y Fernández-Días (2004) encontraron que un profesor valorado como competente por sus estudiantes es aquel que, además de conocer la materia que imparte, se muestra activo y dinámico durante las clases, está atento al alumno, escucha activamente sus dudas y atiende sus problemas. A su vez, es aquel que presenta clases organizadas, siguiendo una estructura lógica, siendo claro en sus explicaciones y ejemplos.

Por otro lado, Merellano-Navarro y otros (2016) hallaron que los alumnos al momento de valorar al buen docente universitario conjugan características pedagógicas, con un enfoque humanista, priorizando la centralidad en el aprendizaje del estudiante. Además, valoran el dominio del contenido con respecto al saber científico y la calidad de la metodología de enseñanza que imparte.

Otras investigaciones realizadas en España (Castro-Morera et al., 2020; De-Juanas Oliva y Beltrán-Llera, 2014; Fernández-Borrero y González-Losada, 2012; Galván-Salinas y Farías-Martínez, 2018) también coincidieron en que los alumnos resaltaban la capacidad del profesor de explicar con claridad, motivar al alumnado, mostrar interés en el aprendizaje de los estudiantes, así como también, de promover la participación activa y la buena comunicación en el aula.

A su vez, varios estudios realizados en Latinoamérica (Andrade-Abarca et al., 2018; Hamer-Flores, 2013; Reyes-Piñuelas et al., 2018; Rocha, 2013) dan cuenta que para los alumnos los aspectos más valorados del quehacer docente son la claridad de las exposiciones, la impartición de clases interesantes, la posibilidad de establecer una relación con los estudiantes que los motive a involucrarse en el aprendizaje y la preparación y organización del curso.

Se cree importante considerar la perspectiva de los estudiantes, ya que ellos son actores fundamentales de los procesos de enseñanza y aprendizaje, y son quienes finalmente van a implementar las mejoras e innovaciones que se propongan (Gargallo-López et al., 2017). Asimismo, es fundamental contar con instrumentos de evaluación adecuados, que garanticen la validez y fiabilidad de sus resultados, y que respondan a las características de la población a la cual se desea evaluar (ITC, 2017).

De acuerdo a lo expuesto, se plantean los siguientes objetivos, en primer lugar, analizar las propiedades psicométricas de fiabilidad y validez del Cuestionario de Competencias Docentes del Profesorado Universitario (CCDPU) para su aplicación en el Área Metropolitana de Buenos Aires (AMBA); y, en segundo lugar, identificar las competencias y atributos mejores valorados por los estudiantes universitarios de Buenos Aires a la hora de evaluar la calidad docente en educación superior.

El CCDPU es un cuestionario diseñado en España por López-Cámara (2014) cuyo objetivo es conocer la opinión que tienen los estudiantes respecto a la calidad de la docencia universitaria. Si bien la versión original está validada en idioma español, resulta pertinente realizar estudios de equivalencia lingüística y revisión de las propiedades psicométricas de una prueba que será aplicada en un contexto distinto al original. Sobre esto, Romero (2011) advierte el impacto que tienen las variaciones lingüísticas, por más sutiles que sean, de los hablantes de un mismo idioma, sobre la validez de contenido y constructo de un test $y$, en consecuencia, en el error de medición.

\section{Método}

\subsection{Diseño}

Se trata de un trabajo de investigación empírico, con enfoque cuantitativo y de alcance descriptivo-correlacional.

\subsection{Muestra y Muestreo}

La muestra del estudio fue generada a través de un muestreo no probabilístico de estudiantes de universidades públicas y privadas. Participaron 120 estudiantes universitarios del Área Metropolitana de Buenos Aires (AMBA), de los cuales 94 fueron mujeres (78,3\%) y 26 hombres $(21,7 \%)$. La media 
de edad fue de 23,57 ( $\mathrm{DE}=4.262$; con un rango de edad entre 19 y 43 años). Por otro lado, de los 120 estudiantes, 63 asistían a universidades privadas (52,5\%) mientras que 57 de ellos concurrían a universidades públicas $(47,5 \%)$. Los alumnos se encontraban cursando diferentes carreras y estaban en distintos años de las mismas.

\subsection{Instrumentos}

1. Encuesta de datos personales y sociodemográficos: se recolectaron los datos mediante un conjunto de preguntas construidas ad hoc orientadas a recabar información acerca de variables sociodemográficas tales como edad, género, carrera de grado en curso, año y universidad a la que se encuentran matriculados.

2. Cuestionario de Competencias Docentes del Profesorado Universitario (López-Cámara, 2014): Se trata de un cuestionario diseñado en España, cuyo objetivo es evaluar la percepción que tienen los estudiantes acerca de la docencia universitaria. Está compuesto por 40 ítems contemplados en seis dimensiones:

a) Diseño de los programas/guías docentes de la asignatura: Planificación detallada de cualquier asignatura que orienta el proceso de enseñanza-aprendizaje. Incluye la selección de contenidos, la estructura y distribución en el programa, criterios para su evaluación y el trabajo del estudiante en torno a ese contenido (ítems: $1 \mathrm{al}$ 7)

b) Metodología docente: Modo de desarrollar la práctica diaria. Responde a cómo se puede proceder con los alumnos para favorecer su formación desde los contenidos de la enseñanza. Pone de manifiesto las intenciones educativas del docente y sus premisas didácticas (ítems: 8 al 22).

c) Actividades prácticas: Diseño y desarrollo de prácticas vinculadas a los contenidos teóricos de las materias de la rotulación para el desarrollo de competencias profesionales (ítems: 23 al 25).

d) Recursos didácticos: Cualquier recurso que el profesorado prevea emplear en el diseño o desarrollo del programa para aproximar y facilitar los contenidos, medir en las experiencias de aprendizaje, desarrollar actividades cognitivas, apoyar sus estrategias metodológicas y facilitar o enriquecer la evaluación (ítems: 26 al 28).

e) Sistemas de evaluación: Procesos sistemáticos de recogida, análisis e interpretación de la información relevante para medir o describir cualquier faceta de la instancia educativa (ítems: 29 al 33).

f) Actitud del profesorado: Creencias, pensamientos e ideas que influyen en la labor y actuación docente (ítems: 34 al 40).

Cada afirmación presentada debe ser contestada a través de una escala tipo Likert de cinco niveles en relación a su importancia para la evaluación de la calidad docente universitaria $(1=$ Totalmente en desacuerdo, 2 = Parcialmente en desacuerdo, 3 = Ni de acuerdo ni en desacuerdo, $4=$ Parcialmente de acuerdo, 5 = Totalmente de acuerdo). Se obtienen puntuaciones parciales de cada una de las dimensiones. Las propiedades psicométricas del instrumento original fueron analizadas y garantizadas por la autora López-Cámara en su trabajo de tesis doctoral (2014).

3. Técnica de Elección de objeto (Martín-Kniep, 2007): Se trata de una técnica que estudia de forma no convencional la práctica docente y tiene como objetivo expresar con mayor complejidad un pensamiento o una idea, recurriendo a lo visual, lo sonoro y lo afectivo. Se les solicita a los participantes la elección de un objeto o una imagen que representase, simbólicamente, al buen profesor. Las respuestas dadas son interpretadas como una metáfora de la evaluación de la calidad docente por cada entrevistado.

\subsection{Procedimiento}

En primer lugar, se solicitó autorización a la autora del CCDPU, Ana Belén López-Cámara, para su adaptación argentina. La recolección de datos se realizó en forma individual, a través de la plataforma virtual ofrecida por Google Forms. Primero se presentó un consentimiento informado solicitando el acuerdo a los individuos de participar en la presente investigación, cuyos resultados serían anónimos 
y utilizados únicamente para los fines de la misma. Luego, se aplicó una encuesta construida ad hoc de datos sociodemográficos y personales (edad, género, carrera de grado en curso, año y universidad a la que asiste) y, por último, se presentó el CCDPU.

Para la validación se realizó un estudio de juicio de expertos con el objetivo de analizar la pertinencia conceptual y lingüística del CCDPU. A su vez, este estudio permitió evaluar la validez de contenido del cuestionario. Para ello se seleccionaron a tres profesores universitarios en materias de psicología, lengua y literatura, a los fines de juzgar de manera independiente los ítems del instrumento. Se les solicitó que completaran una planilla y valoren los criterios de claridad, coherencia, relevancia y suficiencia del CCDPU, siguiendo una escala tipo Likert de 4 niveles ( 1 = "no cumple con el criterio"; 2 = "bajo nivel"; 3 = "moderado nivel"; 4 = "alto nivel"). A su vez, se les facilitó una definición conceptual de cada una de las dimensiones del cuestionario a los fines de lograr mayor precisión en la valoración del contenido del CCDPU

A los efectos de estudiar las propiedades psicométricas de fiabilidad y validez del CCDPU, se utilizó el software estadístico Statistical Package for the Social Sciences (SPSS) versión 24.0 y las plantillas Excel. El análisis de la fiabilidad se realizó a través del análisis de la consistencia interna cuyo indicador es el coeficiente alfa de Cronbach; se analizaron los valores de alfa obtenidos para cada subescala, así como también la correlación total de elementos corregida y las puntuaciones de alfa si cada uno de los ítems es eliminado de las mismas. Asimismo, se llevó a cabo un análisis de correlación entre las dimensiones del cuestionario, mediante el coeficiente $r$ de Pearson, con el objetivo de estudiar la relación entre ellas.

Para el análisis de la validez de criterio, se realizó una entrevista focalizada a 20 estudiantes universitarios que completaron previamente el CCDPU. Se les solicitó que pensaran y describieran a un profesor suyo que consideren que haya sido un buen docente. Además, en el contexto de la entrevista focalizada, se incluyó la técnica de elección de objeto, propuesta inicialmente por Martín-Kniep (2007). Se les solicitó a los participantes la elección de un objeto o una imagen que representase, simbólicamente, al buen profesor. Siguiendo con esta técnica, también se pidió a los entrevistados representar al buen docente con un tipo de música y un sentimiento o una emoción. En una segunda instancia, se efectuó un análisis cualitativo de las entrevistas, a fin de observar concordancias entre los puntajes del CCDPU y las respuestas dadas por los sujetos.

Finalmente, para identificar las competencias y atributos mejores valorados por los estudiantes acerca de la calidad del profesorado universitario se calcularon la distribución de frecuencias y los estadísticos descriptivos básicos para cada uno de los ítems y para las dimensiones que componen el CCDPU. También, se analizó la información cualitativa aportada en la entrevista focalizada y la técnica de elección de objeto.

\section{Resultados}

\subsection{Análisis de las propiedades psicométricas del CCDPU}

\subsubsection{Fiabilidad}

Con respecto a la fiabilidad del CCDPU, en el Cuadro 1 se presentan los índices de fiabilidad calculados a través del Coeficiente alfa de Cronbach para cada una de las subescalas del cuestionario. Siguiendo los criterios de George y Mallery (1995) para la interpretación de los coeficientes de alfa de Cronbach, la puntuación de alfa obtenida para la subescala actitud del profesorado $(\alpha=, 928)$ es considerada excelente; los coeficientes de alfa de las subescalas diseño de los programas/guías docentes de la asignatura $(\alpha=, 824)$, metodología docente $(\alpha=, 887)$, recursos didácticos $(\alpha=0,817)$ y actividades prácticas $(\alpha=, 798)$ son buenos; y para la subescala sistemas de evaluación $(\alpha=0,681)$ la consistencia interna es aceptable. 
Cuadro 1

Estadisticas de Fiabilidad para las subescalas del CCDPU

\begin{tabular}{lcc}
\hline \multicolumn{1}{c}{ Dimensiones del CCDPU } & Alpha de Cronbach & N $^{\circ}$ de elementos \\
\hline $\begin{array}{l}\text { 1. Diseño de programas/guías docentes de la } \\
\text { asignatura }\end{array}$ &, 824 & 7 \\
2. Metodología docente &, 887 & 15 \\
3. Actividades Prácticas &, 798 & 3 \\
4. Recursos Didácticos &, 817 & 3 \\
5. Sistemas de Evaluación &, 681 & 5 \\
6. Actitud del profesorado &, 928 & 7 \\
\hline
\end{tabular}

Nota. Elaboración propia.

Asimismo, se calcularon las estadísticas de la correlación total de elementos corregida para cada una de las subescalas y las puntuaciones de alfa de Cronbach si el ítem es eliminado de las mismas. Los resultados demuestran que todos los ítems presentan una adecuada correlación con la puntuación total (coeficientes mayores a ,30), siendo los ítems 11 (Relación entre los distintos bloques temáticos de la materia) y 31 (Información del sistema de evaluación al finalizar el curso) los de menor puntaje, con ,347 y ,377 respectivamente.

Por otro lado, a los fines de analizar la relación entre las dimensiones del CCDPU, se calcularon las correlaciones entre las puntuaciones directas de cada una de ellas, utilizando el coeficiente r de Pearson. Los resultados indican que existe una relación significativa entre las dimensiones Diseño de los programas/ guía docentes de la asignatura y Metodología docente $(r=, 773 ; \mathrm{p}=, 000)$; Diseño de los programas/guía docentes de la asignatura y Actividades prácticas $(r=, 718 ; \mathrm{p}=, 000)$; Diseño de los programas/guía docentes de la asignatura y Sistemas de evaluación $(r=, 657 ; \mathrm{p}=, 000)$;Diseño de los programas/guía docentes de la asignatura y Actitud del profesorado $(r=, 652 ; \mathrm{p}=, 000)$;Diseño de los programas/guía docentes de la asignatura y Recursos didácticos $(r=, 556 ; \mathrm{p}=, 000)$; Metodología Docente y Actividades Prácticas $(r=, 772 ; \mathrm{p}=, 000)$; Metodología docente y Sistemas de Evaluación $(r=, 691 ; \mathrm{p}=, 000)$; Recursos Didácticos y Metodología Docente $(r=, 601 ; \mathrm{p}=, 000)$;Actividades Prácticas y Sistemas de Evaluación $(r=, 607$; $\mathrm{p}=, 000)$; Actividades prácticas y recursos didácticos $(r=, 510 ; \mathrm{p}=, 000)$; Actividades prácticas y Actitud del profesorado $(r=, 600 ; \mathrm{p}=, 000)$; Metodología Docente y Actitud del Profesorado $(r=, 730 ; \mathrm{p}=, 000)$; Sistemas de evaluación y Recursos didácticos $(r=, 553 ; \mathrm{p}=, 000)$; Sistemas de Evaluación y Actitud del profesorado $(r=, 603 ; \mathrm{p}=, 000)$; Actitud del profesorado y Recursos didácticos $(r=, 517 ; \mathrm{p}=, 000)$. Tal y como se supone teóricamente, las dimensiones del cuestionario covarían positivamente. En el Cuadro 2 se pueden observar las medias y desvíos de las puntuaciones de cada una de las subescalas que componen el cuestionario.

Cuadro 2

Estadísticos descriptivos básicos de las subescalas del CCDPU

\begin{tabular}{llll}
\hline \multicolumn{1}{c}{ Dimensiones del CCDPU } & N & Media & DE \\
\hline 1. Diseño de programas/guías docentes de la asignatura & 120 & 30.47 & 4,46 \\
2. Metodología Docente & 120 & 64,72 & 8,41 \\
3. Actividades Prácticas & 120 & 13,08 & 2,11 \\
4. Recursos Didácticos & 120 & 12,37 & 2,68 \\
5. Sistemas de Evaluación & 120 & 21,12 & 3,37 \\
6. Actitud del Profesorado & 120 & 32,06 & 4,6 \\
\hline
\end{tabular}

Nota. Elaboración propia. 


\subsubsection{Validez}

Para analizar la validez de contenido se sometió a juicio de expertos el contenido del cuestionario. Para ellos se seleccionaron tres docentes universitarios, en materias de psicología, lengua y literatura, a los cuales se les solicitó valorar los ítems del CCDPU según el grado de claridad, coherencia, relevancia y suficiencia, siguiendo una escala tipo Likert de 4 niveles ( 1 = "no cumple con el criterio"; 2 = "bajo nivel"; 3 = "moderado nivel"; 4 = "alto nivel").

Los resultados demuestran que el CCDPU evidencia validez de contenido. La mayoría de los ítems tuvieron medias elevadas (entre 3,5 y 4) en función de la claridad, coherencia y relevancia, lo cual refleja que el contenido de cada subescala del cuestionario resulta pertinente y relevante.A su vez, hubo acuerdo entre el grupo de expertos en que los ítems pertenecientes a cada una de las dimensiones del cuestionario son representativos y suficientes del constructo que pretenden medir, lo cual evidencia la validez de contenido del cuestionario.

Con respecto a la validez de criterio, a partir del análisis cualitativo de las entrevistas realizadas, se observa que lo expresado verbalmente por los estudiantes en sus respuestas, se puede ver reflejado en los puntajes del cuestionario. Así, por ejemplo, uno de los entrevistados, con respecto al docente elegido, señaló "Me gustaba porque explicaba muy bien, primero que nada, se le entendía todo. Y, además, siempre era muy amable con los alumnos. En sus clases integraba a los alumnos, digamos eran muy participativas, y eso hacía que no sea aburrido. Siempre ponía muchos ejemplos para que nosotros entendamos y participemos". Se puede establecer una relación entre dicha respuesta y el cuestionario en los ítems 8 (Organización y presentación de los materiales adecuada -explicaciones, actividades, materiales), 34 (Actitud receptiva en su relación con el alumnado) y 39 (Creación de un clima de trabajo y participación), siendo valorados con una puntuación de 5 (Puntaje más alto) por el entrevistado.

A su vez, cabe destacar que aquellos sujetos que valoraron el uso de recursos didácticos en la entrevista, respondiendo a la pregunta ¿Qué objeto o imagen representaría para usted al buen docente?, con objetos tales como pizarrón, proyector y computadora, puntuaron el ítem 26 (Uso de recursos adecuados -pizarra, transparencias, medios audiovisuales, material de apoyo en red virtual...- que faciliten el aprendizaje) con un puntaje de 5 (puntuación más alta). Por ejemplo, uno de los estudiantes eligió un proyector para representar al buen docente porque "es una herramienta de clase que sirve mucho. También se me ocurrió pizarra, pero proyector me parece que ofrece más posibilidades, es más didáctico.”

Siguiendo estos resultados, otro participante eligió la alegría como emoción para representar a los buenos docentes, y refirió que "los profesores tienen que tener alegría para trasmitírsela a los alumnos, motivarlos y tengan más ganas de estudiar”. Esto puede asociarse al ítem 14 del cuestionario (Fomento de la partición en clase y motivación al alumnado), valorado con un puntaje de 5 por el sujeto. Por su parte, otro entrevistado escogió un libro, señalando que "al asociar un buen profesor al objeto de libro me da a entender que es un profesor que tiene conocimiento, que investiga, que le pone empeño a la clase que está dando", y puntuó el ítem 36 (Interés por la asignatura por parte del docente) también con un 5.

\subsection{Evaluación de la calidad docente según la perspectiva de los estudiantes universitarios}

A fin de responder al segundo objetivo, identificar las competencias más valoradas por los estudiantes universitarios en la evaluación de la calidad docente, se analizaron la distribución de frecuencias y estadísticos descriptivos básicos para cada uno de los ítems que conforman el CCDPU. Los resultados obtenidos evidencian que la mayoría de los elementos que configuran la escala han sido valorados positivamente por el alumnado encuestado. No obstante, cabe señalar algunos comentarios más específicos.

Los ítems 11 (Relación entre los distintos bloques temáticos de la materia), 12 (Coordinación entre el profesorado para evitar solapamientos y relacionar contenidos de distintas asignaturas), 28 (Diseño de cuadernos de prácticas para su implementación en el aula) y 31 (Información del sistema de evaluación al finalizar el curso), presentaron las medias más bajas (entre 3,60 y 3,98). Vale decir que constituyen los aspectos menos relevantes para el alumnado a la hora de evaluar la calidad de la actividad docente.

En el lado opuesto, se ubican los ítems 8 (Organización y presentación de los materiales adecuada), 18 (Exposición de ejemplos en los que se ponen en práctica las competencias a desarrollar por la asignatu- 
ra), 35 (Respeto hacia el alumnado), 36 (Interés por la asignatura por parte del docente), 39 (Creación de un clima de trabajo y participación) y 40 (promoción de una comunicación fluida y espontánea) con las medias más altas, a los cuales alrededor del $70 \%$ de los estudiantes los calificaron con un 5 (puntaje más alto). Se puede decir que para los participantes dichos aspectos incidirán de manera positiva en la labor profesional de un docente universitario.

A su vez, a partir de los resultados, se observa que todos los ítems pertenecientes a la subescala actitud del profesorado fueron calificados mayormente con la puntuación más alta por la muestra de estudiantes encuestados. En relación al Diseño de los programas y guías docente, un 64\% de los estudiantes destacaron principalmente los ítems 2 (Vinculación de los contenidos con los diferentes aspectos de la profesión) y un 70\% el ítem 6 (Adecuación de la bibliografía y los recursos con las finalidades de la asignatura) como los más relevantes.

Con respecto a la subescala Metodología docente, los estudiantes coincidieron que los ítems 8 (Organización y presentación de los materiales adecuada), 9 (Coherencia entre los objetivos y contenidos de la asignatura y las actividades realizadas) y 18 (Exposición de ejemplos en los que se ponen en práctica las competencias a desarrollar por la asignatura) constituyen elementos relevantes en la calidad del profesorado universitario. Estos ítems fueron puntuados con la calificación más alta por más del $65 \%$ de los estudiantes. En segundo lugar, se valoraron los ítems 14 (Fomento de la participación en clase y motivación al alumnado) y 16 (Uso de metodologías para implicar activamente a los estudiantes en el aprendizaje).

Por otro lado, una valoración general de los ítems que constituyen la subescala Recursos didácticos da cuenta que todos son medianamente relevantes, destacando principalmente el uso de recursos, tanto tradicionales como tecnológicos, que faciliten el aprendizaje. Lo mismo ocurre con la subescala Actividades prácticas, en la cual el $68 \%$ de los estudiantes coincidieron que el ítem 25 (Utilidad de las prácticas para el desarrollo de las competencias profesionales) resulta el más importante.

Finalmente, en relación a los Sistemas de evaluación, se observa que el 70\% de los estudiantes entrevistados estuvieron "totalmente de acuerdo" con las afirmaciones de los ítems 29 y 32, dando cuenta que se valora que la información con respecto a los exámenes se dé al comienzo del curso y que ésta sea coherente con los objetivos, contenidos y actividades dadas durante la clase. Con respecto a los demás aspectos de la subescala, se observa mayor variabilidad en las opiniones de los participantes.

Por otro lado, la información cualitativa analizada de las entrevistas y la técnica de elección de objeto da cuenta que los alumnos valoran sobre todo aspectos actitudinales del docente para con sus estudiantes por sobre el conocimiento o las prácticas pedagógicas utilizadas. Esto se puede observar en respuestas tales como "Y el buen docente sería como unas manos abiertas porque como que están abiertos a recibir chicos, personas con dudas e inquietudes, y creo que su misión es de contener y enseñar"; "Era buen profesor porque estaba súper atento al alumnado, si entendíamos o no, que participemos, que demos nuestro punto de opinión" y "Los buenos docentes tienen que ser un soporte para sus alumnos, tipo guiarlos en el proceso de aprendizaje".

\section{Discusión y conclusiones}

Para el desarrollo del presente trabajo se plantearon dos objetivos principales: a) analizar las propiedades psicométricas de fiabilidad y validez del Cuestionario de Competencias Docentes del Profesorado Universitario (CCDPU), diseñado en España por López-Cámara (2014), para su aplicación en el Área Metropolitana de Buenos Aires (AMBA); y b) identificar las competencias y atributos mejores valorados por los estudiantes universitarios de Buenos Aires a la hora de evaluar la calidad de la docencia universitaria. El CCDPU es un cuestionario autoadministrable de 40 ítems cuya finalidad es conocer la opinión de los alumnos universitarios sobre aquellas características que resultan más relevantes en la evaluación docente. Se administró el cuestionario a 120 estudiantes universitarios del AMBA. Los resultados sugieren que el CCDPU presenta evidencias satisfactorias de validez y fiabilidad para su aplicación en Buenos Aires. A su vez, se observó que la subescala mejor valorada por los participantes, como se verá más adelante, fue Actitud del Profesorado, la cual refiere a creencias, pensamientos y formas de actuar y ser del profesor, influyentes en su rol profesional. 
Con respecto al primer objetivo, como se mencionó, los resultados del análisis de las propiedades psicométricas del CCDPU demostraron evidencias aceptables de fiabilidad y validez. La consistencia interna del cuestionario, evaluada mediante el cálculo del coeficiente alfa de Cronbach, se considera aceptable según los criterios propuestos por George y Mallery (1995), con coeficientes superiores a .70 para las subescalas Diseño de los programas/guías docentes de la asignatura $(\alpha=, 824)$, Metodología docente $(\alpha=, 887)$, Actividades prácticas $(\alpha=, 798)$, Recursos didácticos $(\alpha=, 817)$ y Actitud del profesorado $(\alpha=, 928)$. Con respecto a la subescala sistemas de evaluación, la consistencia obtenida fue menor $(\alpha=, 681)$.

Por su parte, la prueba original diseñada por López-Cámara (2014), presentó valores de alfa similares a los del presente estudio. Fueron más elevados para las subescalas Diseño de los programas/guías docentes $(\alpha=, 864)$, Metodología docente $(\alpha=, 915)$, Actividades prácticas $(\alpha=, 813)$ y Sistemas de evaluación $(\alpha=, 851)$. No obstante, para las subescalas Recursos didácticos y Actitud del profesorado, la consistencia obtenida fue menor, con coeficientes de alfa a de ,811 y 0,906 respectivamente.

En relación a las evidencias de validez, el análisis de contenido del CCDPU reflejó niveles aceptables de acuerdo entre los expertos encuestados. La mayoría de los ítems fueron calificados como coherentes y pertinentes, constituyendo una muestra representativa de la subescala en la que se encuentran incluidos. Asimismo, se presentaron evidencias aceptables de validez de criterio, ya que se pudieron observar concordancias entre los resultados del cuestionario y las respuestas dadas por los estudiantes en las entrevistas realizadas. Así, por ejemplo, se evidenció que aquellos alumnos que destacaron el diseño y uso de materiales y recursos didácticos como relevantes en la función docente, valoraron positivamente los ítems incluidos en la subescala Recursos didácticas del CCDPU.

Siguiendo esta lína, resulta importante llevar a cabo estudios de validación de un instrumento de origen distinto al argentino, por más que el idioma sea el mismo. Romero (2011) señala que, aunque varias culturas compartan el mismo idioma como en el caso de Argentina y España, los significados de las mismas palabras pueden varía notablemente, así como también la frecuencia de uso y el grado de familiarización de distintas palabras. No obstante, en el presente estudio no se encontraron dificultades en la comprensión de la consigna ni de los ítems del cuestionario.

Por otro lado, para responder al segundo objetivo, se llevó a cabo un estudio descriptivo a fin de conocer la perspectiva de los estudiantes sobre la calidad docente. Los resultados de la aplicación del CCDPU a la muestra de 120 estudiantes universitarios, demostraron que los ítems pertenecientes a la subescala Actitud del profesorado fueron valorados positivamente por la mayoría de los participantes. De esto se deduce que, desde la perspectiva de los alumnos, los buenos docentes son aquellos que se muestran receptivos y respetuosos con sus estudiantes, buscan motivarlos, se interesan activamente por su proceso de aprendizaje y logran crear un clima adecuado de trabajo y participación durante las clases.

En segundo lugar, se valoraron dos aspectos relacionados a la Metodología docente, por un lado, los alumnos destacaron que el buen profesor es quien presenta clases organizadas, da explicaciones adecuadas del contenido de la materia y utiliza ejemplos y recursos que faciliten el aprendizaje de los alumnos. Y, por otro lado, se apreciaron elementos más interpersonales tales como el fomento de la participación en clase, el desarrollo de la capacidad de síntesis y razonamiento del alumnado, y el desarrollo de habilidades relacionadas con la práctica profesional.

Los resultados obtenidos en este estudio son similares a lo expuesto en investigaciones anteriores. Lizasoain y otros (2017) hallaron que los estudiantes relacionan la calidad docente con tres ejes principales: transmisión de motivación e interés en el proceso de aprendizaje de los alumnos, explicaciones de los contenidos claras y ordenadas, y actitudes de comprensión, empatía y atención. Por su parte, Fernández-Borrero y González-Losada (2012) encontraron que los alumnos consideraban que el uso de estrategias didáctico-pedagógicas, así como también características personales como la paciencia y el respeto, resultan aspectos más importantes a la hora de evaluar la calidad del profesorado universitario que el conocimiento específico de su área del saber.

Por otro lado, los hallazgos de la presente investigación reflejan la demanda por parte de los estudiantes de orientar los procesos educativos hacia un modelo centrado en el aprendizaje y el alumno. En estos últimos años, se habla de un cambio de paradigma educativo, en el cual el alumno y su aprendizaje toman mayor protagonismo y, en consecuencia, el rol de profesor universitario adquiere nuevas competencias y desafíos (Sayós et al., 2014). Es por ello que los alumnos tienden a valorar como mejor competentes 
a aquellos docentes que facilitan la participación del estudiante en la clase, la comunicación eficaz, el desarrollo de capacidades de deducción y pensamiento crítico, y promueven aprendizajes colaborativos.

Cabe considerar algunas limitaciones de la presente investigación. En primer lugar, el tamaño pequeño de la muestra $(n=120)$ limita su representatividad, por lo tanto, se debe tener en cuenta que se trata de un estudio preliminar. En segundo lugar, la validación del cuestionario se llevó a cabo con estudiantes universitarios del AMBA, por lo cual resultaría necesario constatar los resultados del presente estudio en las diferentes regiones de Argentina. Por otro lado, el análisis de la validez de criterio debió realizarse mediante un estudio cualitativo a través d entrevistas mixtas, debido a que no se cuentan con otros instrumentos sobre el tema validados en Argentina.

No obstante, el análisis de las entrevistas realizadas evidencia que los puntajes del cuestionario coinciden con lo expuesto verbalmente por los estudiantes. Por lo tanto, se puede decir que el CCDPU demuestra evidencias aceptables de validez de criterio. A su vez, a partir de las respuestas de los alumnos entrevistados, se observó que los estudiantes definen a los buenos profesores como aquellos que muestran interés en el proceso de aprendizaje de los alumnos y actitudes de respeto, comprensión y amabilidad para con ellos, motivando a los estudiantes a participar, expresar sus opiniones y dudas libremente y favoreciendo una comunicación fluida en el aula. Además, se valoró la vocación y el interés que demuestra los buenos docentes para enseñar y por la materia que imparten

Dada la tendencia de los estudiantes a valor aspectos actitudinales de los profesores por sobre aspectos metodológicos y científicos, resultaría interesante pensar en variables asociadas a rasgos de personalidad de los docentes, como por ejemplo la empatía, y estudiar sus influencias en la calidad de la actividad docente, y por consecuente en la formación de los futuros profesionales.

Sobre la base de lo señalado, se concluye que, en primer lugar, el CCDPU presenta evidencias suficientes de validez y fiabilidad para su utilización en el contexto argentino y, por lo tanto, resulta una herramienta útil para futuras investigaciones sobre el tema. El cuestionario permite identificar las competencias más relevantes a la hora de evaluar la calidad de la docencia universitaria, según la perspectiva de los estudiantes. En segundo lugar, se concluye que las siguientes competencias fueron las mejores valoradas por los estudiantes: el desarrollo de un adecuado clima de trabajo, la promoción de la participación y el intercambio, la motivación del alumnado, la planificación docente, la claridad en las explicaciones y la utilización de diversos recursos que faciliten el aprendizaje.

\section{Referencias}

Acevedo-Álvarez, R. y Fernández-Díaz, M. J. (2004). La percepción de los estudiantes universitarios en la medida de la competencia docente: validación de una escala. Revista Educación, 28(2), 154-166.

https://doi.org/10.15517/revedu.v28i2.2257

Andrade-Abarca, P. S., Ramón-Jaramillo, L. N. y Loaiza-Aguirre, M. I. (2018). Aplicación del SEEQ como instrumento para evaluar la actividad docente universitaria. Revista de Investigación Educativa, 36(1), 259-275.

https://doi.org/10.6018/rie.36.1.260741

Castro-Morera, M., Navarro-Asencio, E. y Blanco-Blanco, Á. (2020). La calidad de la docencia percibida por el alumnado y el profesorado universitarios: análisis de la dimensionalidad de un cuestionario de evaluación docente. Educación XX1, 23(2), 41-65. https://doi.org/10.5944/educXX1.2571

Coppola, N. L. (2012). La evaluación de la función docente en la Universidad de Buenos Aires [Comunicación]. Simposio Pensar la universidad en sus contextos: Perspectivas evaluativas Universidad Nacional de Río Cuarto, Buenos Aires, Argentina.

Cortés, E., Campos, M. y Moreno, M. (2014). Priorización de las dimensiones de evaluación al desempeño docente por el estudiante, en tres áreas del conocimiento. Formación Universitaria, 7(2), 3-10.

https://doi.org/10.4067/S0718-50062014000200002

De-Juanas Oliva, Á. y Beltrán-Llera, J. A. (2014). Valoraciones de los estudiantes de ciencias de la educación sobre la calidad de la docencia universitaria. Educación XXI, 17(1), 57-82.

https://doi.org/10.5944/educxx1.17.1.10705 
Fernández-Borrero, M. A. y González-Losada, S. (2012). El perfil del buen docente universitario. Una aproximación en función del sexo del alumnado. Revista de Docencia Universitaria, 10(2), 237-249.

https://doi.org/10.4995/redu.2012.6106

Galván-Salinas, J. O. y Farías-Martínez, G. M. (2018). Características personales y práctica docente de profesores universitarios y su relación con la evaluación del desempeño. Revista Iberoamericana de Evaluación Educativa, 11(2), 9-33. https://doi.org/10.15366/riee2018.11.2.001

Gargallo-López, B., Pérez-Pérez, C., Verde-Peleato, I. y García-Félix, E. (2017). Estilos de aprendizaje en estudiantes universitarios y enseñanza centrada en el aprendizaje. Revista Electrónica de Investigación y Evaluación Educati$v a$, 23(2), art2. https://doi.org/10.7203/relieve.23.2.9078

George, D. y Mallery, P. (1995). SPSS/PC + Step by step: A simple guide and reference. Belmont.

Hamer-Flores, A. (2013). La percepción del buen profesor en alumnos de nuevo ingreso a la enseñanza universitaria: El caso de ETEA (Córdoba). Revista Complutense de Educación, 26(2), 227-240. https://doi.org/10.5209/rev_RCED.2015.v26.n2.41534

International Test Commission. (2017). The ITC guidelines for translating and adapting tests. https://www.intestcom.org/page/16

Lizasoain, L., Etxeberria, J. y Lukas, J. F. (2017). Propuesta de un nuevo cuestionario de evaluación de los profesores de la Universidad del País Vasco. Estudio psicométrico, dimensional y diferencial. Revista Electrónica de Investigación y Evaluación Educativa, 23(2), art1. http://doi.org/10.7203/relieve.23.2.10436

López-Cámara, A. B. (2014). Diseño de un protocolo de evaluación de las competencias docentes del profesor universitario. Tesis Doctoral, Universidad de Córdoba, España.

Loureiro, S., Míguez, M. y Otegui, X. (2016). Desempeño docente en la enseñanza universitaria: análisis de las opiniones estudiantiles. Cuadernos de Investigación Educativa, 7(1), 55-67. https://doi.org/10.18861/cied.2016.7.1.2576

Luna, E. y Torquemada, A. (2008). Los cuestionarios de evaluación de la docencia por los alumnos: balance y perspectivas de su agenda. Revista Electrónica de Investigación Educativa, Especial, 10, 1-15.

Martín-Kniep, G. (2007). Portfolios del desempeño de maestros, profesores y directivos: la sabiduría de la práctica. Paidós.

Martínez, J. (2013). Combinación de mediciones de la práctica y el desempeño docente: consideraciones técnicas y conceptuales para la evaluación docente. Pensamiento Educativo, Revista de Investigación Educacional Latinoamericana, 50(1), 4-20. https://doi.org/10.7764/PEL.50.1.2013.2

Martínez, D., Gaetan S., Romero J., Saavedra M. y Alvarado, P. (2005). Caracterización de la práctica docente universitaria. Estudio de casos: Pregrado. Tesis de Maestría, Universidad Javeriana, Colombia.

Merellano-Navarro, E., Almonacid-Fierro, A., Moreno-Doña, A. y Castro-Jaque, C. (2016) Buenos docentes universitarios: ¿Qué dicen los estudiantes?. Educação e Pesquisa, 42(4), 937-952.

https://doi.org/10.1590/s1517-9702201612152689.

Monereo, C. y Pozo, J. I. (2003) La universidad ante la nueva cultura educativa: enseñar y aprender para la autonomía. Síntesis.

Reyes-Piñuelas, E. P., González-Machado, E. C. y Be-Ramírez, P. A. (2018). Evaluar la práctica docente a partir de los comentarios de estudiantes universitarios. Revista Iberoamericana de Educación, 76(2), 117-134. https://doi.org/10.35362/rie7623096

Rocha, R. (2013). Escala de opinión de los estudiantes sobre la efectividad de la docencia (EOEED) en educación superior. Formación Universitaria, 6(6), 13-22. https://doi.org/10.4067/S0718-50062013000600003

Romero, E. (2011). Confiablidad y Validez de los instrumentos de evaluación neuropsicológica. Subjetividady Procesos Cognitivos, 15(2), 83-92.

Romero-Díaz, T. (2014). Cuestionario de opinión para la evaluación del desempeño docente en la Unan-Managua, Nicaragua, Farem-chotales. Revista Actualidades Investigativas en Educación, 14(2), 1-28.

https://doi.org/10.15517/aie.v14i2.14804 
Salazar-Gómez, E. y Tobón, S. (2018). Análisis documental del proceso de formación docente acorde con la sociedad del conocimiento. Revista ESPACIOS, 39(53), 17-30..

Sayós, R., Pagés, T., Amador, J. A. y Jorba, H. (2014). Ser buen docente ¿Qué opinan los estudiantes de la Universidad de Barcelona? Revista Iberoamericana de Psicología y Salud, 5(2), 135-149.

Spooren, P., Vandermoere, F., Vanderstraeten, R. y Pepermans, K. (2017). Exploring high impact scholarship in research on student's evaluation of teaching (SET). Educational Research Review, 22, 129-141.

https://doi.org/10.1016/J.EDUREV.2017.09.001

Tangney, S. (2014). Student-centred learning: a humanist perspective. Teaching in Higher Education, 19, 266-275. https://doi.org/10.1080/13562517.2013.860099

\section{Breve CV de las autoras}

\section{Mariana Soledad Seivane}

Licenciada en Psicología por la Pontificia Universidad Católica Argentina (UCA). Medalla de oro de la Facultad de Psicología y Psicopedagogía, UCA. Becaria de Iniciación a la Investigación en el Centro de Investigaciones en Psicología y Psicopedagogía (CIPP), UCA. Sus principales líneas de trabajo son "Desarrollos psicométricos en inteligencia y personalidad" $\mathrm{y}$ "Estudios acerca del procesamiento psicológico del tiempo en adultos", sobre las cuales ha presentado varias ponencias en congresos y jornadas de intercambio académico e investigación. Profesora asistente de "Técnicas de Evaluación Psicológica I”, carrera de Psicología, en la Facultad de Psicología y Psicopedagogía, UCA. Evaluadora en el Departamento de Ingreso y Departamento de Orientación Preuniversitaria, UCA. Email: mariana_seivane@uca.edu.ar

ORCID ID: https://orcid.org/0000-0002-9162-6935

\section{María Elena Brenlla}

Doctora en Psicología por la Pontificia Universidad Católica Argentina (UCA) y Licenciada en Psicología por la Universidad de Buenos Aires (UBA). Diploma de honor de la Facultad de Psicología, UBA. Profesora titular de "Metodología de la Investigación” y de “Técnicas de Evaluación Psicológica I”, Carrera de Psicología, Facultad de Psicología y Psicopedagogía, UCA. Investigadora y directora de líneas de investigación ("Diseño y adaptación de tests"; "Estudios acerca del procesamiento psicológico del tiempo en adultos") en el Centro de Investigaciones en Psicología y Psicopedagogía (CIPP), UCA. Profesora de Posgrado (Doctorado) en las Facultades de Psicología y Psicopedagogía y de Ciencias Económicas y Sociales de la UCA. Profesora de Posgrado (Maestría) en la Facultad de Psicología de la UBA y en la Facultad de Psicología de la Universidad Nacional de La Plata. Ex asesora psicométrica en la Secretaría de Evaluación Educativa, Ministerio de Educación de la Nación. Actualmente realiza la especialización en Entornos Virtuales de Aprendizaje (SIIED, UCA). Email: bren@uca.edu.ar

ORCID ID: https://orcid.org/0000-0003-2536-9499 